\title{
Ultrasonography in GCA assessment
}

Although colour duplex ultrasonography (CDS) is increasingly accepted as a diagnostic tool for giant cell arteritis (GCA), this imaging modality is still not widely used in routine clinical practice, owing largely to the need for skilled sonographers. A retrospective analysis of CDS findings in 293 patients with suspected or confirmed large-vessel vasculitis suggests CDS could reduce the need for temporal artery biopsy (TAB) - currently the gold standard for diagnosing GCA, but an invasive procedure - and also provides support for the role of CDS in the detection of disease flares in follow-up.

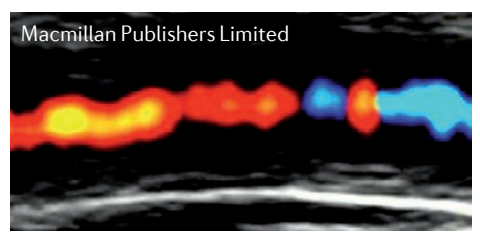

Monti and colleagues used a CDS protocol based on previous evidence and initially developed and tested as part of the Temporal Artery Biopsy vs Ultrasound in Diagnosis of GCA (TABUL) study, with standardized methods of sonographer training and interpretation of CDS findings. The 293 consecutive patients referred to a fast-track outpatient clinic between July 2014 and September 2016 were assessed clinically by a rheumatologist and underwent CDS of the temporal arteries and axillary arteries at each visit.

Among the 210 patients with suspected GCA, a diagnosis was clinically confirmed in 118 . CDS findings were positive in 52 of these 118 (44\%) cases. Notably, CDS had a sensitivity of $63.3 \%$ and a specificity of $100 \%$ in new referrals treated with glucocorticoids for $<7$ days; among those treated with glucocorticoids for $>7$ days, the specificity remained high (98.3\%) but sensitivity dropped to $43.6 \%$. In patients with jaw claudication and high inflammatory markers, sensitivity rose to $81.8 \%$.

The rate of $\mathrm{TAB}$ declined over the observation period, from 72 (42\%) in 2014-2015 to 36 (25\%) in 2016 $(P=0.002)$, which the authors suggest reflected increased recognition that positive CDS provided sufficient evidence of GCA. CDS was also able to detect changes in 'halo' distribution during follow-up and could be useful in detecting disease flares.

Sarah Onuora

ORIGINAL ARTICLE Monti, S. et al. The proposed role of ultrasound in the management of giant cell arteritis in routine clinical practice. Rheumatology (Oxford) http://dx.doi.org/10.1093/rheumatology/ kex341 (2017)

FURTHER READING Dejaco, C. et al. Giant cell arteritis and polymyalgia rheumatica: current challenges and opportunities. Nat. Rev. Rheumatol. 13, 578-592 (2017)

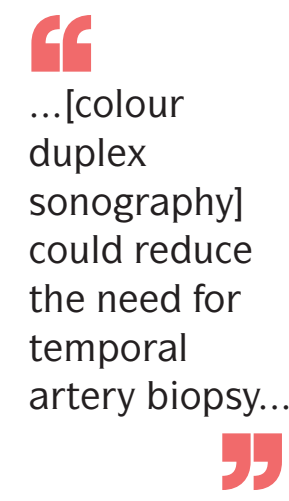

